Cahiers de recherches médiévales siècle)

\title{
Des lettres de nouvelles au temps de la guerre d'Italie de Charles VIII (avril-mai 1495)
}

\section{Marion Pouspin}

\section{(2) OpenEdition \\ Journals}

Édition électronique

URL : https://journals.openedition.org/crm/11734

DOI : $10.4000 / \mathrm{crm} .11734$

ISSN : 1955-2424

Éditeur

Honoré Champion

Édition imprimée

Date de publication : 20 novembre 2009

Pagination : 459-478

ISSN : $1272-9752$

Référence électronique

Marion Pouspin, " Des lettres de nouvelles au temps de la guerre d'Italie de Charles VIII (avril-mai 1495) », Cahiers de recherches médiévales [En ligne], 18 | 2009, mis en ligne le 15 décembre 2012, consulté le 15 décembre 2022. URL : http://journals.openedition.org/crm/11734 ; DOI : https:// doi.org/10.4000/crm. 11734 


\title{
酶RM
}

\section{Des lettres de nouvelles au temps de la guerre d'Italie de Charles VIII (avril - mai 1495)}

\begin{abstract}
Letters written from Naples between April-May 1495 by nobles serving in the army of Charles VIII constitute a privileged source for analysing the role of news-conveying letters in shaping relationships within a social network. These letters bear witness, in both content and form, to a bond of "amicitia" based on the circulation of mutual services. Each epistolary document devises two approaches: an offer of information and a request for a favor. The transmission of news of the military situation as regards the troops or the health of acquaintances always implies a petition for a favor or a kindness. The disclosure of the information is then the compensation for the request. In anthropological terms, the letter is conceived, certainly, as a representation of the writer to his correspondents, but also as an instrument for strengthening a network of friendship within which social relationships are organized.
\end{abstract}

Résumé: Des lettres rédigées entre avril-mai 1495 à Naples par des gentilshommes de l'armée de Charles VIII constituent un terrain d'enquête privilégié pour l'analyse des enjeux de la lettre de nouvelles dans les relations interpersonnelles au sein d'un réseau social. Ces missives adoptent le type de relation de l'amicitia qui se fonde sur la circulation de prestations réciproques et qui se traduit ici dans la forme même des lettres. Dès lors, chaque document épistolaire combine deux démarches : offrir une information et sommer un bienfait. La transmission de nouvelles sur la situation militaire des troupes et sur l'état de santé des hommes implique toujours une supplique d'un bienfait moral ou matériel. La divulgation de l'information est alors la contrepartie, d'une loyauté égale, à la requête. En termes anthropologiques, la lettre est conçue, certes, comme une présentification du destinateur auprès de ses correspondants, mais également comme un instrument pour un réseau d'amitié renforcé en lequel s'ordonne la sociabilité du groupe social.

«Monsieur mon pere, à vostre bonne grasse me recommande, sans oblier madamoyselle ma mere. Des nouvelles de par dessa, nous somes dedans Naples et fasson bonne chiere, et croit que le roy ne demorera guere de par desse. Il est grand bruit tous les jours que yl doit partir [...]. Les gens de la Chambre du roi ont fait la fete dans Naples; j'ai prit part et ai debourser dix ecus que j'avais empruntés pour cela; il faut les rendre à la femme du preteur, Madame de Saint Verne [...]. Le plus toust qui cera posible, m'en retourneray au pays $[\ldots] »^{1}$. Voici comment un certain «J. Chabane», gentilhomme accompagnant Charles VIII dans son expédition italienne, adresse à son père une lettre de nouvelles rédigée le 8 mai 1495 à Naples ${ }^{2}$.

\footnotetext{
${ }^{1}$ A. Cutolo, «Nuovi documenti francesi sulla impresa di Carlo VIII », Archivio storico per le provincie napolitane, 63, 1938, p. 21.

${ }^{2} \mathrm{Ce}$ «J. Chabane» renvoie sans doute à Jacques II de Chabannes, maréchal de La Palice et gentilhomme de la maison du roi, qui a encouragé les projets du roi d'expédition en Italie et qui a participé à ce voyage au cours duquel il se distingua à Fornoue, le 6 juillet 1495.
}

Cahiers de Recherches Médiévales, 18, 2009 
Cet extrait met en évidence l'instrumentalisation de la lettre dans les relations interpersonnelles au sein d'un réseau social par l'articulation de deux fonctions: informer et demander. Cette instrumentalisation se traduit par la stylisation du langage pensée en fonction de l'identité du destinataire, stylisation que codifient les artes dictandis tels que celui de Boncompagno da Signa en $1215^{3}$.

Certaines catégories de lettres ont bénéficié, depuis quelques décennies, d'analyses très fines sur la nature des informations qu'elles colportent, telles que les missives des étudiants à leurs parents ou à leurs proches rendant compte de la difficulté de leurs études ou de besoins pécuniaires pressants, les lettres de marchands ou de banquiers adressées à un compatriote dans lesquelles se négocie la communication de conseils et de renseignements sur les fluctuations du marché ou encore les lettres échangées entre membres d'une même famille . Quant aux « lettres de nouvelles échangées par des amis en temps de guerre», il n'est plus nécessaire aujourd'hui de montrer l'ampleur des informations qu'elles diffusent et leur apport à

Dans cet article, le terme de «gentilshommes » sera préféré au mot «combattants » pour désigner les destinateurs de ces lettres en référence à leur statut social et en raison de l'absence de récits de batailles dans leurs missives, absence s'expliquant par la situation de l'armée française au moment où celles-ci sont rédigées. L'occupation de la cité napolitaine induit en effet la suspension des velléités et, par conséquent, celle de la participation des gentilshommes. La contribution de nos destinateurs à la campagne militaire est néanmoins attestée par les lettres elles-mêmes : l'écuyer dit Bosquin (A. Cutolo, op. cit., lettre p. 17-18) précise servir en tant qu'homme d'armes dans la compagnie du comte de Montpensier, Gilbert de Bourbon, gouverneur de Paris en 1494. De même, Adrien de L'Hospital est un capitaine de cent lances (Ibid, lettre p. 24-29). Nous savons par ailleurs qu'il a conduit l'avant-garde lors de la bataille de Saint-Aubin-du-Cormier, l'été 1488, et qu'il a combattu héroïquement à Fornoue.

${ }^{3}$ Boncompagno est né à Signa, près de Florence, entre 1165 et 1175 ; il décède à Florence après 1240. Il a enseigné la grammaire et la rhétorique à l'université de Bologne. On lui doit notamment six traités consacrés aux formules de salutation épistolaire, dont le Boncompagnus en 1215. Sur son œuvre de théorisation de l'art épistolaire, voir V. Abbruzzetti, «La codification de l'art épistolaire au Moyen Âge. Un exemple italien: Boncompagno da Signa », Epistulae Antiqua. $n^{\circ}$ 2. Actes du IIe colloque "Le genre épistolaire antique et ses prolongements européens », Université François-Rabelais, Tours, 28-30 septembre 2000, éd. L. Nadjo et E. Gavoille, Louvain, Paris, Peeters, 2002, p. 367-377.

${ }^{4}$ Sur les lettres des étudiants, voir tout particulièrement l'article de C. H. Haskins, «The Life of Medieval Students as illustrated by their Letters, The American Historical Review, 3, 1897 1898, p. 203-229 [art. revu et corrigé dans les Studies in Medieval Culture, New York, 1929, rééd. 1965, p. 1-35]. Sur les échanges épistolaires entre marchands, voir notamment l'article de J. Hayez, «La gestion d'une relation épistolaire dans les milieux d'affaires toscans à la fin du Moyen Âge ", La circulation des nouvelles au Moyen Âge, XXIVe Congrès de la SHMES, Avignon, juin 1993, Paris, 1994, p. 63-83. Parmi l'abondante littérature sur les lettres échangées entre membres d'une même famille, voir N. Davis, Paston Letters and Papers of the fifteenth century, Oxford, 2004, 2 vol. : C. L. Kingsford, The Stonor Letters and Papers (1290-1483), Londres, 1919, 2 vol. 
l'écriture de l'histoire événementielle ${ }^{5}$. Ainsi la lettre de Jean Sarrasin à Nicolas Arrode corrobore et précise le récit de Joinville sur la prise de Damiette $(1249)^{6}$; la correspondance des croisés (Anselme de Ribemont, Étienne de Blois, Aymeric d'Antioche, Conrad III, etc.) est précieuse pour l'histoire de la croisade ${ }^{7}$; les missives d'une délégation envoyée à Ascalon en 1100 témoignent du massacre de Jérusalem lors de la conquête de la Ville Sainte par les croisés ainsi que de l'effort général entrepris par les chefs de la communauté juive de l'Égypte pour sauver leurs coreligionnaires rescapés de ce massacre ${ }^{8}$.

La richesse de l'information contenue dans ces lettres est presque un lieu commun mais il convient de pousser un peu plus loin l'exploration des enjeux de l'information. Dans cette perspective, la centaine de lettres privées rédigées par des gentilshommes accompagnant Charles VIII dans son expédition italienne (14941495), aujourd'hui conservées aux Archives de Milan, permettent de saisir la valeur instrumentale de l'information et de la lettre. Cette documentation n'a pas encore fourni, de manière générale, un intérêt sur les rapports interpersonnels tissés au sein d'un réseau social. En 1938, publiant des extraits de cette liasse de lettres, Alessandro Cutolo s'intéresse avant tout à l'identification des couples de correspondants dans l'optique d'une analyse sociale et réduit ces documents épistolaires au statut de mines de renseignements divers : événements, rumeurs, vie quotidienne, etc. Récemment, Sara Fourcade s'est appuyée sur quelques-unes des lettres de gentilshommes éditées par A. Cutolo, qu'elle intègre à un corpus épistolaire plus large, dans la perspective de cerner les caractéristiques de la pratique épistolaire de la noblesse laïque au tournant des XV-XVI ${ }^{\mathrm{e}}$ siècles ${ }^{9}$. Si S. Fourcade expose clairement les caractéristiques formelles, matérielles et thématiques de ces lettres dont l'unité réside dans le statut social des destinateurs, elle n'aborde que rapidement l'échange épistolaire en termes de sociabilité : la «nouvelle» politique, militaire ou familiale permet de maintenir le dynamisme de la relation épistolaire nouée entre les correspondants.

Il convient de souligner que, globalement, les missives des gentilshommes ne sont nullement des lettres « inutiles» entre des correspondants n'ayant rien à se demander, rien de pratique à communiquer, visant seulement à «faire plaisir ». Après l'analyse des modalités de rédaction et de transmission des lettres en question, suivie de l'identification de la nature des informations qu'elles divulguent, il s'agit

\footnotetext{
${ }^{5}$ Les lettres échangées entre des amis en temps de guerre constituent l'un des types d'échanges épistolaires définis par Boncompagno: "De litteris quas amici tempore guerre aduinicem sibi transmittunt. », Boncompagnus, livre 6, chap. 3 ; reproduit par L. Rockinger, Briefsteller und Formelbücher des 11. bis 14. Jahrhunderts, Munich, 1969, t. 1, p. 138.

${ }^{6}$ Lettres françaises du XIII ${ }^{e}$ siècle. Jean Sarrasin. Lettres à Nicolas Arrode (1249), éd. A. L. Foulet, Paris, 1924.

7 «Letters of the Crusaders Written from the Holy Land», D. C. Munro, Translations and Reprints from the Original Sources of European History, Philadelphie, 1900, vol. 1, 4, p. $1-40$.

${ }^{8}$ S. D. Goitein, "Contemporary Letters on the capture of Jerusalem by the Crusaders », Journal of Jewish Studies, Londres, Jewish Chronicle Publications, 1952, vol. 3, p. 162-177.

${ }^{9} \mathrm{~S}$. Fourcade, «La pratique épistolaire de la noblesse française au temps des guerres d'Italie (1495-1525), Cahiers de recherches médiévales, Paris, 13, 2006, p. 133-145.
} 
de montrer, à partir de l'exemple de quelques-uns de ces documents, que ces missives recouvrent des enjeux bien concrets se traduisant dans leur forme ellemême.

Les lettres de gentilshommes d'avril - mai 1495 : des témoignages sur le vif d'une actualité politique effervescente

Les tribulations des lettres de gentilshommes : de la cite napolitaine aux archives de Milan

La centaine de lettres des gentilshommes de l'armée de Charles VIII, rédigées en langue vernaculaire avec des sentences latines insérées ci et là, relève des archives de Ludovic Sforza ${ }^{10}$. Ce ne sont pas des lettres diplomatiques, mais de simples lettres privées dont les auteurs décrivent, dans une certaine mesure, la situation de l'armée française à Naples entre avril et mai 1495. Adressées à des correspondants demeurés en France, ces missives ont été interceptées en Lombardie par les hommes du Sforza. En effet, la conquête du royaume de Naples par Charles VIII a été rapide et facilitée par les rivalités entre les États italiens. Cependant, très vite, dès novembre 1494, Ludovic proteste contre l'alliance franco-florentine et devient l'organisateur d'une ligue dont les efforts visent à mettre en échec l'expédition royale. Dès le 12 avril 1495, une Ligue de contre-offensive anti-française se réunit autour de Venise, du pontife, du roi des Romains, des rois d'Espagne et du duc de Milan Ludovic. Ludovic le More cherche alors à amasser toute information sur la date du départ des troupes françaises. Après un séjour napolitain d'un peu plus de trois mois, le retour du roi de France et de son armée devient de plus en plus pressant face à cette Ligue qui, disait-on, rassemble des troupes afin d'empêcher le voyage de Charles VIII et de le faire prisonnier.

La captation des courriers à l'investigation de Ludovic ne se résume sûrement pas à cette centaine de lettres. Cette pratique est explicitement attestée par un combattant de l'armée de Charles VIII dans une lettre rédigée le 15 juillet 1495 et mise en imprimé dans une «pièce d'actualité politique » française :

«Depuis que le seigneur Ludovic eut clos et empeschié le passage de la poste. Et vous ay escript par deux fois C'est assavoir de Sene [Sienne] et de Pize par des courriers qui par mer alloient à Lyon. Ne scay si avez receu les lettres [...]. Mais les dites [lettres], Ludovic a retenu toutes les postes. Et pour ce que maintenant la poste peut aller seurement en France par ceste course de poste vous ay bien voulue escripre ces presentes pour vous faire scavoir voz nouvelles vous advisant que desire bien savoir comment vous portez. $»^{11}$.

Cette pratique d'ennemis - politique ou militaire - ou de concurrents commerciaux s'efforçant à se procurer des nouvelles afin d'obtenir des révélations

\footnotetext{
${ }^{10}$ Cette liasse de lettres est conservée aux Archives de Milan sous la cote Cart. 250.

${ }^{11}$ Cette lettre de Gilbert Pointet nous est parvenue sous sa forme imprimée dans la «pièce d'actualité politique» française intitulée Lettres nouvelles. Datées $d u$ XV. Jour de Juillet escriptes à Ast, Paris, Pierre Le Caron, 4 ff.n.c., in-4º goth., juillet 1495 [Bibl. Mun., Nantes, 40988 (26), recueil factice INC 115].
} 
sur des événements politiques, militaires ou économiques, est bien connue ${ }^{12}$. Dans le cadre de la campagne italienne menée par Charles VIII, cette pratique n'est nullement ponctuelle, comme en témoignent les plaintes concordantes des destinateurs aux prises avec le pillage de la poste qui les opprime. Ces plaintes, récurrentes dans les lettres des gentilshommes, sont également partagées par le roi comme l'atteste cette missive datée du 20 juin 1495 et expédiée au duc de Bourbon :

«Le vous ay souvent escript et faict responce, mais les postes ont esté, comme savez, destroussés et mes lettres retenues, par quoy elles n'ont pu aller jusques à vous, et à ceste cause $\mathrm{j}$ 'ay faict donner ordre que on vous envoyra doresnavant ce qui sera necessaire. $\gg .^{13}$

«Vous m'escripvés que n'avés nulles lettres de moy, don je suis bien esbay, car je vous asseure, que je vous en ay escript bien souvant, mais il a esté destroussé beaucoup de poste en le duché de Myllan, de ceux qui vont en Fransse. $»^{14}$

Ce motif postal constitue un lieu commun de l'ensemble de la production épistolaire, tant médiévale que moderne ou contemporaine. Il donne lieu à des développements convenus. La plainte traduit le désagrément, voire la souffrance, engendré par le retard ou le silence qui hypothétique gravement les certitudes au regard de leurs amis ou parents.

\section{Origine et modalités de transmission des lettres de gentilshommes}

La plupart des souscriptions de ces missives nous éclairent sur l'identité des destinateurs, et les salutations ou suscriptions sur celle de leurs correspondants. Les destinateurs appartiennent globalement à l'entourage royal. Parmi les identifiables, on note des écuyers des écuries royales, des fourriers, un sommelier de paneterie des trésoriers, le maréchal de France, un capitaine de cent lances, à ceux-ci s'ajoutent des seigneurs et des agents royaux, à savoir des trésoriers et un garde du sceau de la ville de Condrieu. Quant à leurs correspondants, Alessandro Cutolo a enquêté sur leur statut à partir des noms mentionnés dans les conclusions des lettres. On discerne des officiers de l'Hôtel du roi et du Parlement de Paris, tels que des fourriers, des écuyers, des notaires et secrétaires, un grenetier, un argentier et maître de la chambre aux deniers de Jeanne de Bourbon, un chevaucheur de l'écurie du roi, un bonnetier du roi, des conseillers au Parlement de Paris, le grand maître des enquêtes et général des monnaies, auxquels s'ajoutent des agents royaux d'administration locale, tels que des prévôts, le gouverneur de Bourgogne, un vicomte, un receveur, etc. Étant donné la certaine homogénéité des statuts sociaux des couples de correspondants, on pourrait envisager ces échanges épistolaires comme des lettres certes privées mais in officio, d'autant plus que les lieux de résidence des destinataires ou d'envoi des lettres se confondent bien souvent avec la géographie de la cour et des autres lieux du pouvoir, notamment Moulins et Paris.

${ }^{12}$ Y. Renouard, «Information et transmission des nouvelles », L'histoire et ses méthodes, dir. C. Samaran, Belgique, 1961, p. 95-142.

${ }^{13}$ Lettres de Charles VIII, roi de France, éd. P. Pélicier, Paris, 1902, t. 4 «1494-1495», p. 218.

${ }^{14}$ A. Cutolo, op. cit., p. 60. 
Pourtant, ces gentilshommes s'adressent à leurs destinateurs en termes de parenté - «ma femme», «mon coussin », «mon frere», «mon pere », «ma seur »ou d'affection - «mon amy», «ma mye»-, combinant parfois deux formules d'adresse tout en ne désignant qu'un unique destinataire, telles que «mon frere et cousin ». S'il est vrai qu'à la fin du Moyen Âge, les termes d'adresse empruntent souvent au vocabulaire adelphique, notamment au sein de relations fortes d'amitié, les notices de dictionnaires de la noblesse corroborent l'idée d'un échange épistolaire unissant, de manière privée et ex officio, des membres d'un réseau social basé essentiellement sur la parenté ${ }^{15}$.

Toutefois, trois lettres relèvent d'un autre type de correspondance. Tout d'abord, Jacques Terris, commis de l'argentier du roi, adresse deux lettres in officio, l'une à Jacques Audebert, chevaucheur des écuries royales, et l'autre à Aubert Durant, bonnetier du roi, tout deux demeurant à Paris ${ }^{16}$. Ces deux lettres mettent en lumière ce que taisent les comptes des argentiers, documents financiers et administratifs, à savoir les mécanismes d'approvisionnement et la pratique de la gestion des vêtements dans le contexte particulier d'un prince en déplacement ${ }^{17}$. Enfin, la lettre du marchand Philibert Gay à son père l'informe des méventes de ses « chemises » à Naples et de son projet d'attendre le départ du roi pour Rome afin de profiter de la sécurité offerte par le convoi de l'armée royale face aux brigands ${ }^{18}$. De là, il projette de se rendre à Pise où son commerce pourra prospérer. Ce marchand, expatrié en Italie, « déplie» sa marchandise de ville en ville. Cela atteste que, à la fin

\footnotetext{
${ }^{15}$ Sur les termes d'adresse calqués sur l'expression de l'affection entre frères et soeurs, voir D. Lett, Famille et parenté dans l'Occident médiéval, $V$-XVe siècle, Paris, 2000, p. 208.

Quant aux principaux dictionnaires de la noblesse consultés pour cet article qui, certes anciens, demeurent précieux pour leurs notices détaillées : J.-C. Bévy (abbé de), Dictionnaire adalphique et chronologique de la noblesse, 1779-1790, 4 t. [Arch. Nat., AB XIX 690, 691, 692, 693]; La Chenaye-Desboys et Badier, Dictionnaire de la noblesse, Paris, 1863-1876. ; Dictionnaire de biographie française, dir. J. Balteau, M. Barroux, M. Prevost et J.-C. Roman d'Amat, Paris, 1933-1989.

${ }^{16}$ L'argentier du roi est chargé, à l'origine, de l'achat de vêtements, de meubles et d'objets précieux ainsi que de veiller à la préparation des cérémonies.

${ }^{17}$ Dans la première lettre, Jacques Terris informe le chevaucheur de la livraison partielle de bonnets et de bottes dont le roi a nécessité : sur les douze bonnets baillés par le bonnetier, seuls six sont parvenus en Italie. Il l'exhorte alors à expédier six autres bonnets que le bonnetier va à nouveau lui transmettre. Ensuite, dans la seconde lettre Jacques Terris demande au bonnetier de bailler les six bonnets au chevaucheur en question (A. Cutolo, op. cit., p. 76).

Les documents traditionnels relatifs à l'argenterie, essentiellement des comptes répertoriant l'ensemble des dépenses de l'office, révèlent la finesse de la budgétisation. Même si le ressort de l'argentier du duc de Bretagne ou de Bourgogne équivaut en quelque sorte à celui de la Chambre des deniers en France, les trois comptes de l'argentier de Charles le Téméraire pour les années 1468-1470 renseignent sur la comptabilité des «menues parties », c'est-à-dire ces sommes versées à tels marchands pour l'achat de marchandises telles que des chausses et chaussons, des draps, des robes, etc. Comptes de l'argentier de Charles le Téméraire duc de Bourgogne, dir. W. Paravicini, Paris, 2002, 2 vol.
}

${ }^{18}$ A. Cutolo, op. cit., p. 22. 
du $\mathrm{XV}^{\mathrm{e}}$ siècle, les marchands itinérants n'ont pas disparu et ce malgré l'essor des grandes compagnies commerciales telles que celles italiennes et hanséates, celles des Stapler anglais, des Fugger d'Augsbourg, etc. Toutefois, ce Philibert Gay ne semble pas un marchand isolé : il correspond avec son père auquel il promet de «faire du mieux possible» pour son commerce, ce qui dénote une agence commerciale familiale. Quant à l'envoi de ce courrier, Philibert Gay entend bénéficier de la rapidité et de la précision du circuit d'échanges dont jouit le roi pour la transmission des nouvelles ${ }^{19}$. Néanmoins, ces courriers se trouvent parfois retardés, voire empêchés par le contexte militaire, comme c'est le cas ici.

Ces lettres de gentilshommes sont envoyées par «paquet», au-dessus desquels, précise l'un des destinateurs, se trouve une «petite liée [...] faisant mention du contenu du dit pacquet». Leur expédition se décline en trois modes différents. Soit elles sont directement adressées et expédiées à leur destinataire. Soit un correspondant unique est mandaté de rapporter oralement les nouvelles contenues dans la lettre auprès de l'entourage proche ou des individus indiqués par le destinateur. Le recours à un correspondant porte-voix d'une missive à destination différée est une pratique commune à d'autres lettres, telles que celle d'Étienne de Blois à Adèle, celle de Jean Sarrasin à Nicolas Arrode ${ }^{20}$, celle de Robert d'Artois à Blanche de Castille datée du 23 juin $1249^{21}$. Soit elles sont adressées à une personne chargée de les transmettre à leurs véritables destinataires :

«Je puis qu'il vous plaise trouver façon de envoyer de pacquet de lettres que je vous envoye à Amboise à Julan Clotet, l'un de mes compagnons [...]. Ces lettres devront, ensuite, parvenir à ma femme. $»^{22}$.

« Je vous adresse une lettre en vous priant de la faire suivre à son destinataire. ${ }^{23}$

«Mon pere ne reçoit pas les lettres que je lui envoie par l'intermediaire de Melly, à Moulines. Voudriés vous, desormais, vous charger de les lui transmettre ?».24

Si des religieux portent des lettres de gentilshommes vers le royaume de France, les missives sont le plus souvent acheminées par les «chevaucheurs» de la

\footnotetext{
${ }^{19}$ Si l'emploi de courriers commerciaux, expédiés généralement par les navires marchands, n'est pas étranger à la pratique commerciale, les marchands profitent d'ordinaire d'un service de poste particulier dont bénéficie la diplomatie ou la politique, plus rapide et plus certain. Les lettres des marchands vénitiens du Levant du XVI siècle témoignent de cette réalité selon laquelle leurs propres moyens de transmission de nouvelles restent inadaptés à leurs nécessités, les conduisant ainsi à recourir à un service de poste plus précis. Sur ce point, voir en particulier l'ouvrage de U. Tucci, Lettres d'un marchand vénitien : Andrea Berengo (15531556), Paris, 1957.

${ }^{20}$ «Faites savoir ces lettres à touz nos amis », cité dans les Lettres françaises du XIII siècle, op. cit., p. 9.

${ }^{21}$ «Hic est tenor literae missae reginae Franciae, verbo ad verbum», lettre reproduite par D. C. Munro, op. cit., lettre ${ }^{\circ} 2$.

${ }^{22}$ A. Cutolo, op. cit., p. 18-19.

${ }^{23}$ Ibid, p. 36.

${ }^{24}$ Ibid, p. 39.
} 
«poste», établie en France depuis le règne de Louis XI sur l'inspiration des exemples milanais et vénitiens ${ }^{25}$.

\section{nouvelles}

Le départ du roi et des troupes françaises : entre rumeurs et

Les gentilshommes décrivent à leurs correspondants la situation de l'armée française en Italie, une armée dont le retour est retardé et reconduit de semaine en semaine. Ces documents reflètent alors les tribulations de l'information militaire du moment, entremêlant nouvelles véritables et rumeurs. Dans ces missives, la rumeur se distingue aisément de la nouvelle par des termes ou expressions spécifiques renvoyant à l'une ou à l'autre. Le mot «rumeur » n'apparaît jamais dans les lettres de gentilshommes, mais il est dénoté par le terme «bruit»- «commun bruyt est que», «il est tout commun que» ou le «grant bruyt que»-, qui est employé pour désigner le contenu de l'information tout en mettant l'accent sur son colportage, par bouche à oreill $\mathrm{e}^{26}$. De façon générale, les destinateurs, tout en rapportant la rumeur derrière un paravent impersonnel et anonyme - «l'on dit que », «ay ouy dire » ou «il est bruit que»-, se tiennent à distance de celle-ci en émettant un avis à propos de sa vraisemblance - «je suis d'oppinion que», «je croy que». Quant au terme «nouvelle», récurrent dans les lettres, il recouvre deux significations. D'une part, il désigne, de façon générale, le contenu de l'information. Forgée sur le terme latin nuovus, la nouvelle annonce «ce qui survient en dernier », un « fait récent» et, par extension, renseigne sur l'état de santé d'une personne. Le mot s'inscrit alors dans l'expression «la nouvelle de ». D'autre part, il renvoie au médium, c'est-à-dire à l'agent colporteur de l'information réduisant celle-ci à un objet et devenant synonyme de lettre d'information ou de «nouvelles à la main $»^{27}$. De surcroît, la rumeur serait plutôt du côté de l'incertain - une nouvelle sans certitude - comme le dénote l'étymologie du mot rumor tandis que la nouvelle serait du côté de la vérité ou de la vraisemblance. Cette distinction est nette dans la citation suivante : « L'on a dit icy que le roy d'Espaigne estoyt mort; touteffoys le roy n'en a point seu de nouvelles sures (pléonasme? $) »^{28}$.

Si le Sforza a pu trouver dans ces missives une certaine satisfaction à son dessein d'obtenir des informations sur le retour imminent du roi de France et de ses troupes, le moment du départ du roi semble toujours se situer du côté de la rumeur :

« Le bruyt commun est que le roy s'en partira d'icy pour tout le moys de may pour s'en retrouver en France [...]. ». ${ }^{29}$

\footnotetext{
${ }^{25}$ «Depuis quinze jours en ça vous ay escript bien au long par ung moyne qui s'en va par della, qui y doit estre environ le X du moys de juing prouchain. », «Je vous ai écrit, un moine vous portera la lettre $»$, Ibid, p. 73-74.

${ }^{26}$ Claude Gauvard fait l'état des lieux de la recherche sur la rumeur dans son article « Rumeur et stéréotypes à la fin du Moyen Âge ", La circulation des nouvelles au Moyen Âge, op. cit., p. 158-177.

${ }^{27}$ Voltaire, Siècle de Louis XIV, Paris, 1864, chap. 26, p. 288.

${ }^{28}$ A. Cutolo, op. cit., p. 48.

${ }^{29} \mathrm{Ibid}$, p. 10.
} 
«Il est ung grant bruyt que le Roy s'en part pour le plus tart d'ici à juit jours, dont nous sommes tous fort joyeux. $\gg .^{30}$

« [Je] croy que [le roi] partira pour se mettre sur son retour au plus loing dedans trois jours ou quatre. $» .^{31}$

Le statut de la rumeur concernant la date du départ du roi est ambigu : la date est, de manière générale, rapportée. Elle relève du bruit, ce qui traduit une mise à distance de l'information. Pourtant, l'annonce de la date semble émaner du roi. Si le médiéviste se heurte bien souvent au silence des sources susceptibles de lui fournir une analyse sur l'origine et sur les mécanismes de la formation de la rumeur, l'une des lettres nous apprend que l'information provient des «paiges » - pages - du roi, selon lesquels «le roy s'en part dedans huit jours pour s'en aller en France $»^{32}$. Ailleurs, l'information vient directement du roi qui « tyent tousjours [ses troupes] de demain en demain $\gg{ }^{33}$.

Plus la date de rédaction des lettres se rapproche de la fin du mois de mai, plus le scepticisme grandit parmi les troupes quant à l'imminence du retour :

«On dit tous les jours que partirons demain, mais demain ne vient point, dont il nous desplait [...] [après l'hommage des Napolitains au roi, nous] partirons [...] et sera lundi, quod non credo, car on l'a tant dit que nous ne le crerons jusques à ce que le voyons. $\gg .^{34}$

«Au demorant, l'ont dit que nous partiron demain ou mardy, mes je le croiré quant nous serons à vingt lieux d'icy $»{ }^{35}$

«Croys que [le voyage de retour] ne sera de long temps car le roy baille tousjours des bourdenes et dit de VIII jours en VIII jours tout ainsi qu'il nous a amené $»{ }^{36}$ «Le roy a prit son propos de partir lungdi ou mardi. Je en scay qui l'en fera. ». ${ }^{37}$

«Nous sommes sur nostre partement de nous aller de jour en jour : passés sont XV jours, mais il ne peut plus retarder que nous en soyons en chemin. ${ }^{38}$

«Le roy, à l'aide de Dieu, espere se partir d'ici de Napples pour s'en aller en Franse d'ici quatre ou cinq jours mes je n'en scay rien au vray et je cuide que ne sait il aussy luy, et pour autant, ne vous tenés point à malaise sy on dit plusieurs paroles d'aller de retourner, car plusieurs en parlent et n'en scavent rien. $»{ }^{39}$

La rumeur quant au moment du départ du roi et des troupes naît de l'écart entre ce qui est dit et ce qui est cru, écart nourri par des bruits qui cristallisent des inquiétudes. Ces inquiétudes sont issues d'une tension entre l'expectative du départ

\footnotetext{
${ }^{30}$ Ibid, p. $18-19$.

${ }^{31}$ Ibid, p. 54-56.

${ }^{32}$ Ibid, p. 19.

${ }^{33}$ Ibid, p. 58-59.

${ }^{34}$ Ibid, p. 45.

${ }^{35}$ Ibid, p. 45-46.

${ }^{36} \mathrm{Ibid}$, p. 56-57.

${ }^{37}$ Ibid, p. 60-61.

${ }^{38}$ Ibid, p. 63.

${ }^{39}$ Ibid, p. 63-64.
} 
de l'armée et les empêchements retardant sa concrétisation, à savoir l'attente d'ambassades envoyées auprès du pape afin de connaître son intention face à l'entreprise de Charles VIII, le mariage de Ligny ${ }^{40}$, ainsi que l'entrée solennelle de Charles VIII à Naples. Cette tension est accentuée par des bruits et des nouvelles concernant la situation militaire de l'armée de Charles VIII en Italie : le départ des troupes françaises est perçu comme de plus en plus pressant en raison d'une part, de l'organisation d'une contre-offensive italienne renforcée par des contingents espagnols et, d'autre part, de la dangerosité croissante du paysage et du climat italiens :

«Le bruyt commun est que [...] toutes les Italles, tant le pape, les Fleurantins, les Genevoys, les Venyciens, le duc de Mylan, se sont alliés ensemble contre nous. $\gg .^{41}$ (Annexe)

«Au regart des Ytalles, nous ne les creignons gueres, et croy, à mon advis, qu'il nous lesserons voulemptiers passer en ne leur demendent riens; et touchant le Duc de Millan, il en est icy grand bruyt de son armée qu'il a faicte, touteffoys, nous n'en tenons pas grant compte. $\gg{ }^{42}$

«On nous a dit que les Veniciens sont descendus in magno numero pres d'icy. Credo tamen istud fabulosum. On a dit aussi que le roy de l'Espaigne a pris les biens $\mathrm{du}$ roy Alfonse pro data sue sororis et nepotum. Illud etiam est ridiculum dictum. $\gg .{ }^{43}$

"Il est grant bruit qu'il [le Pape] nous veult empescher les passaiges. ». ${ }^{44}$

«Est nouvelle qu'il [le roi] partira sans nulle faulte d'icy à deux ou troys jours pour s'en retorner, et aussi est besoing qu'il s'en aille ou aultrement nous serions ycy toux brulex car c'est ung horrible chouse de la grande chaleur qu'il y fait, et aussi de l'autre part, il y a tous les ans aux villes de par deça mortalité. »». ${ }^{45}$

Cette mise à distance de ces informations par les gentilshommes est très intéressante: de là, la nouvelle émanant directement du roi ou par l'intermédiaire des pages devient rumeur. La rumeur est « un phénomène qui commence à se faire jour sous l'effet d'un regard contrasté», elle provient d'une tension, d'un heurt" Dans cet écart, on peut saisir la «psychologie collective», autrement dit les croyances et les sentiments tels que la certitude en la force du roi de France face aux Italiens et aux Espagnols - cette foi en la supériorité du roi traduit le sentiment national des gentilshommes - et les inquiétudes face à la dangerosité du paysage et du climat italiens. Ces témoignages de l'expérience, que sont les lettres de gentilshommes, se nourrissent des nouvelles officielles équivoques et dénaturées : ces croyances et ces sentiments trahissent non pas la "psychologie individuelle»,

\footnotetext{
${ }^{40}$ «Et [si] ne furent les nopces de monsieur de Liney, qui ont esté ceste sepmaine passée, nous feussions ja partiz. », Ibid, p. 73-74.

${ }^{41}$ Ibid, p. 10.

${ }^{42}$ Ibid, p. 18-19.

${ }^{43}$ Ibid, p. 24.

${ }^{44}$ Ibid, p. 52.

${ }^{45}$ Ibid, p. 56-57.

${ }^{46}$ É. Remy, «Comment saisir la rumeur?», Ethnologie française, 23, 1993, p. 591-602.
} 
mais bien la «psychologie collective» de laquelle «relève surtout la fausse nouvelle» (Marc Bloch $)^{47}$.

\section{La lettre de nouvelles et son instrumentalisation}

Au delà des révélations sur la situation des troupes françaises que Ludovic a cherchées à acquérir, les gentilshommes communiquent à leurs proches des informations d'une toute autre nature, relevant d'enjeux bien concrets. Grâce à l'écriture épistolaire, l'éloignement et le contexte de la guerre ne dénaturent pas les relations entre le couple d'amis. En témoigne la concordance entre le type d'information délivrée et l'identité du destinataire visé. Cela éclaire, comme le souligne S. Fourcade, la capacité des gentilshommes «à personnaliser le discours épistolaire $\aleph^{48}$. Autrement dit, on n'adresse pas les mêmes propos à son père, à sa mère, à sa sœur, à sa femme ou encore à son cousin ou seigneur, etc. À qui écrivent ces gentilshommes? Parmi les lettres ou extraits de lettres publiés par Alessandro Cutolo, plus de la moitié environ sont destinées à un membre de la cellule familiale, soit $25 \%$ à un frère, $13 \%$ à l'épouse, $10 \%$ à un ami non spécifié, $9 \%$ au père, $5 \%$ à la mère, $4 \%$ à une sœur et également au cousin, enfin $1 \%$ à un oncle. Les autres missives sont dépêchées pour $13 \%$ auprès d'officiers royaux, compagnons des destinateurs, pour $6 \%$ auprès de leur seigneur, $4 \%$ à un trésorier, $6 \%$ à un ecclésiastique, une veuve, un gouverneur ou un avocat sans que la nature de leurs relations ne puisse exactement être appréciée.

Quant au contenu des propos développés, il faut rappeler que les lettres privées «naissent presque toujours d'occasions qui sont des besoins $»^{49}$ : la lettre est un moyen d'obtenir du destinataire un bienfait, qu'il soit moral ou matériel. Ainsi, la composition d'une lettre recouvre deux démarches, combinées bien souvent au sein d'une même lettre : la lettre, ou plutôt une partie de la lettre, qui consiste à offrir et la lettre, ou partie de lettre, qui consiste à demander.

\section{La transmission de nouvelles comme prestation d'un bienfait}

De la première démarche relève la circulation de l'information relative à l'expédition italienne ou à l'état d'une personne. Si l'annonce du retour imminent des troupes françaises est une constante dans l'ensemble des lettres de gentilshommes, un seul événement fait l'objet d'une description. Il s'agit de la cérémonie de l'entrée de Charles VIII à Naples le 12 mai 1495, qui constitue d'ailleurs le seul événement, au sens de fait mémorable, survenu entre les mois d'avril et de mai :

\footnotetext{
${ }^{47}$ M. Bloch, Réflexions d'un historien sur les fausses nouvelles de la guerre, Paris, 1999, p. 17. À partir de son expérience personnelle et de quatre ouvrages relatifs à la psychologie du soldat, Marc Bloch s'intéresse à la genèse et au développement des fausses nouvelles nées et colportées au cours de la Première Guerre Mondiale. Pour leur part, les lettres de nouvelles des gentilshommes rendent compte que la Guerre d'Italie menée par Charles VIII (14941495) a été, comme toute guerre, une «immense expérience de psychologie sociale».

${ }^{48}$ S. Fourcade, «La pratique épistolaire... », op. cit., p. 140.

${ }^{49}$ D. J. Leclercq, «L'amitié dans les lettres au Moyen Âge. Autour d'un manuscrit de la bibliothèque de Pétrarque », Revue du Moyen Âge latin, Lyon, 1, 1945, p. 402.
} 
«Le roy feist mardi dernier son entrée en ceste ville, qui fut en robe et non en armes, et estoit habillé en habit royal, portant la couronne en la teste, et tenant une pomme d'or ronde et ung septre ès mains. Ses officieux principaulx du pays estoient habillés comme est le chancelier en France, et le grant chambellan, qui estoit monsieur le senechal de Beauquere portoit son espée roialle. La dicte entrée ne fut pas si belle que selle de Florence, la plus belle que je veiz oncques. $\gg .^{50}$

«Le roy, à qui Dieu doint bonne vye et longue, feist son entrée en ceste ville mardi dernier, vestu d'une robbe de drap d'or doublée de semblable, tenant en l'une de ses mains ung ceptre et en l'autre une pome d'or, affeublé d'une tocque noire, et la coronne dessus, faicte en ceste ville. Devant lui estoient les sires de Montpensier, Vy-roy de ce royaume de Sicile, le senechal de Beaucaire, gouverneur et grant chambellan, qui portoit l'espée d'honneur, de Precy, grant seneschal et le prince de Salerne, admiral du dit royaume, tous vestuz de robbes d'escarlate, chascun ung bonnet d'escarlate à grant rebraz sur la teste. fourrez de letisses; lesquelz sembloient mieulx president que aultres gens. Devant eulx estoient les archers de la garde et les arbalestriers, tout à pyé, chascun son hocqueton vestu, et devant eulx, l'eglise. Apres le roy estoient les gentilz hommes de pyé, vestuz de leurs robes de nopces, leurs haches au poing seulement. Apres qu'il eus testé parmy la ville, il vint en la grant eglise où il feist plusieurs chevaliers, monsieur de Dunoys, le premier, et plusieurs autres seigneurs, ensemble le senechal de Lyonnois, Rigaut de Reille, Peron de Bacher, le prevost de l'ostel, Torquet, et plusieurs autres, et sur le soir en son logeis de Capouane il receupt les homaiges. ».. ${ }^{51}$

Au moyen de ces descriptions détaillées, les lettres présentent un tableau de l'événement. Elles font tableaux. Ces représentations linguistiques donnent à voir l'événement aux destinataires en leur mettant sous les yeux son image absentec'est ce qui définit l'ekphrasis selon Daniel Arasse ou l'hypothytose. Les lettres offrent, font part du tableau de l'événement au même titre que l'une des 117 figures coloriées de la Cronaca napoletana figurata du chroniqueur Ferraiuolo ${ }^{52}$.

\footnotetext{
${ }^{50}$ Ibid, p. 45.

${ }^{51}$ Ibid, p. 50-51.

${ }^{52}$ Dans sa chronique, le napolitain Ferraiuolo relate les événements de la prise et de l'occupation de Naples vus de l'intérieur et assortit son récit de figures coloriées qui illustrent les différentes phases de la conquête française dont il a été le témoin oculaire. La figure $\mathrm{n}^{\circ} 44$ ( $\mathrm{d}$ 'après le manuscrit 801 de la bibliothèque Perpont-Morgan de New York) est la traduction graphique de sa description de l'entrée de Charles VIII à Naples : «E lo Re de Franza veneva in miezo de ditta compagnia, quale veneva sotte uno palio de inborcato de oro, che lo portavano li gintili homini delli Siegi. Et Sua Maiestà a cavallo ad una cavalla chiamata Belladonna, et guarnita de inborcato. Et Sua Maistà portava vistuto una roba de inborcato d'oro, et un mano manca portava una palla de oro piccola et alla mano ritta portava la bacchetta d'ariento [...]. ». Texte transcrit et figures reproduites dans Una Cronaca napoletana figurata del quatrocento, dir. R. Filangieri, Naples, 1956, p. 133.
} 
Néanmoins, les destinateurs ne revendiquent pas la valeur documentaire de leur description, mais entendent procurer du «plaisir» par la lecture de telle nouvelle ${ }^{53}$.

Autant dire que l'essentiel de l'information délivrée se concentre sur l'état de santé des destinateurs qui offrent ainsi un bienfait moral ou spirituel à leurs correspondants. En effet, cela devait les satisfaire de la même manière que la réception de nouvelles similaires réjouit les gentilshommes.

Les missives adressées aux femmes délivrent encore un autre type d'information, à savoir la formulation de sentiments affectifs à l'égard de la lectrice :

« Je vous asseure que je ne vous oblive point. ${ }^{54}$

«La chose que je desire le plus, c'est de vous veoir, : j'ay mis et mectray peine de ce faire le plus tost qu'il me sera possible. $» .^{55}$

«Plust à Dieu que seussiés bien la fin [sic] que j'ay de vous voir, et sy vous an anquestés [quand] seront della, vous verrés se que l'on vous an dira. $\gg .^{56}$

Ce motif de la plainte devant l'éloignement de la personne aimée n'est pas propre aux lettres de gentilshommes. Des formules d'amour analogues se retrouvent dans des Artes Dictaminis, tels que les Lettres de deux amants et les Lettre d'amour du «manuscrit de Tegernsee», si bien que l'on pourrait douter de la spontanéité et de la sincérité de l'expression de tels sentiments et y voir plutôt l'application d'un art épistolaire connu de tout homme cultivé ${ }^{57}$. Cependant, comme le démontre Étienne Wolff, ces lettres ont une «base authentique». Les collecteurs de recueils épistolaires rassemblent des lettres d'amour auxquelles ils ôtent tout contenu personnel afin de les rendre anonymes et leur donner valeur générale de modèle ${ }^{58}$. Si les rares correspondances amoureuses suivies dont nous disposons se trouvent à la suite d'un traité épistolaire, des lettres indépendantes, dont l'authenticité ne fait aucun doute, corroborent la thèse d'Étienne Wolff. Ainsi, en 1098, alors à Jérusalem, Étienne, comte de Blois et de Chartes, adresse une lettre à son épouse

\footnotetext{
${ }^{53}$ « Je vous porteray de nouvelletez de deça où, à mon advis, prendrez plaisir», A. Cutolo, op. cit., p. 15 .

${ }^{54}$ Ibid, p. 10.

${ }^{55}$ Ibid, p. 38-39.

${ }^{56}$ Ibid, p. 73.

${ }^{57}$ Le manuscrit 1452 de la Bibliothèque de Troyes contient une centaine de lettres échangées entre deux amants (Héloïse et Abélard ?), dans l'une desquelles l'homme manifeste son désir de rencontrer la dame: "Precor te obnixe, ut michi mandes, quomodo te habeas, quia tua prosperitas est mea summa voluptas. Manda michi, quando venire possim. Vale. » (fol. 164 ${ }^{\mathrm{r}}$ ) [ «Je te supplie instamment de m'écrire comment tu vas, car ton bien-être est mon plus grand plaisir, fais moi savoir aussi quand je pourrai venir. Porte-toi bien. »]. E. Könsgen, Epistolae duorum amantium, Briefe Abeleards und Heloises ?, Cologne, 1974, p. 38, lettre n ${ }^{\circ} 68$.

Quant au «manuscrit de Tegernsee», les dix lettres illustrent les préceptes contenus dans les traités théoriques. Traduction et présentation de ces lettres par É. Wolff, La lettre d'amour au Moyen Âge, Paris, 1996, p. 93-118.

${ }^{58}$ Ibid, p. 27. Outre son Boncompagnus, Boncompagno da Signa a également écrit une Rota Veneris qui modélise la correspondance amoureuse selon les étapes de la relation entre les deux amants.
} 
Adèle dans laquelle il exprime sa volonté de la revoir le plus tôt possible ${ }^{59}$. Ce même empressement des retrouvailles de l'être aimé, véritable topos des lettres d'amour médiévales, se retrouve également dans les missives de femmes à leur amant, telles que celles adressées par la dame de Remiremont à Pierre de Hagenbach dans la seconde moitié du $\mathrm{XV}^{\mathrm{e}}$ siècle $^{60}$. En bref, tout en respectant les préceptes de la rhétorique épistolaire et en s'inscrivant dans le moule formel prescrit, l'expression des sentiments des gentilshommes éclaire un pan de l'histoire de la vie privée délicat à saisir : les sentiments échangés au sein d'un couple ${ }^{61}$.

\section{La petitio et la supplique d'un bienfait}

Dans les missives des gentilshommes, la transmission de l'information s'accompagne toujours d'une requête. Ces suppliques sont de deux sortes. D'une part, elles consistent souvent en des biens spirituels qui résultent de la réciprocité de la nouvelle sur l'état de santé. Ce souci d'avoir ce type de dépêches apparait comme une préoccupation majeure, voire obsessionnelle :

«Désirant savoir de voz nouvelles, car depuis que receuz vos lettres, je vous ay escript deux fois, dont n'ay eu aucune réponse de vous. $\gg{ }^{62}$

« Je vous prie que les sollicitez à m'escripre plus souvent car c'est le plus grant plaisir que j'aye que d'en savoir. $»{ }^{63}$

«Pourquoy ne m'escrivez vous pas plus souvent? $»{ }^{64}$

L'exemple d'Antoine de la Tour, écuyer du roi, qui a pris «aise», au sens d'état agréable, d'avoir des nouvelles de son amie est représentatif de cette soif d'informations procurant des bienfaits moraux ${ }^{65}$.

${ }^{59}$ «You will certainly see me just as soon as I can possibly return to you. Farewell.», «Letters of the Crusaders Written from the Holy Land », D. C. Munro, op. cit., lettre n`2.

${ }^{60}$ A partir d'un corpus de onze lettres authentiques qu'adresse la dame de Remiremont à Pierre de Hagenbach à la fin du $X V^{\mathrm{e}}$ siècle, Werner Paravicini a tenté de reconstruire le dialogue écrit entre ces deux amants. Après une enquête minutieuse d'identification de cette voix féminine, W. Paravicini explore la nature des liens que noue cette correspondance, ainsi que l'expression des sentiments et les tendres formules ainsi échangées. Que ce langage soit conventionnel ou celui du cœur, ces lettres comme le démontre W. Paravicini, sont idoines à contribuer à une histoire des émotions. W. Paravicini, «Un amour malheureux au $\mathrm{XV}^{\mathrm{e}}$ siècle : Pierre de Hagenbach et la dame de Remiremont », Journal des Savants, janvier-juin 2006, p. $105-181$.

${ }_{61}$ Précieuse pour l'analyse des sentiments exprimés au sein couple est l'intense correspondance du marchand Francesco de Prato. Ces échanges épistolaires conjugaux, débutés en 1382 au moment du premier départ de Francesco pour Pise, nous fournissent une «image très coloriée » de la vie quotidienne, conjugale et familiale au XIV ${ }^{\mathrm{e}}$ siècle. Sur ce point, voir l'ouvrage d'I. Origo, Le marchand de Prato. La vie d'un banquier toscan au XIV siècle, trad. J. Fillion, Paris, 1959.

${ }^{62}$ A. Cutolo, op. cit., p. 35-36.

${ }^{63} \mathrm{Ibid}$, p. $38-39$

${ }^{64}$ Ibid, p. 39.

${ }^{65}$ Ibid, p. 39-40. 
D'autre part, des services matériels sont sollicités. Tandis que les requêtes précédentes concernent l'ensemble des lettres et sans distinction du genre du destinataire, ces pétitions de services matériels semblent propres aux correspondances échangées entre un père et son fils, un officier et son seigneur ou unissant deux frères ${ }^{66}$. En effet, leurs prières concernent soit la donation d'un office ou d'une provision en France ou en Italie, soit la recherche d'un office vacant ${ }^{67}$, voire même la requête de régler en France une affaire conclue en Italie. Ainsi Louis le Laye demande à son père, Guillaume de Laye, seigneur de Saint Ligier, de rembourser la somme de quatre-vingt livres tournois à la femme de l'écuyer Jean Amiot, dit Bousquin, qui lui a fourni, en Italie, un cheval ${ }^{68}$. L'écuyer lui-même prie son seigneur Guillaume de Laye de rembourser à sa femme la somme de ce cheval ${ }^{69}$.

Cette préoccupation d'acquérir un bienfait, spirituel ou matériel, se traduit dans la forme des lettres, puisque successivement il s'agit, en théorie, de saluer celui à qui l'on s'adresse - la salutatio -, de capter sa bienveillance - la captatio benevolentiae -, d'exposer l'objet de la lettre - la narratio - et la requête - la petitio - et, enfin, de conclure par les remerciements anticipés - la conclusio ${ }^{70}$. Dans la pratique, un écart s'opère entre les modèles offerts par les dictatores, maîtres en ars dictaminis, qui sont bien connus dans toute l'Europe à la fin du Moyen Âge, et la correspondance rédigée par ces gentilshommes. La composition de leurs lettres se limite à une salutation brève, se résumant à la mention des noms de l'auteur et du destinataire, à une narration elle aussi brève, à une requête et à la souscription.

Conformément aux règles formulées par les maîtres épistoliers, les traits formels d'une lettre sont adoptés et plus encore le type de relation, l'amicitia, censé fonder l'échange épistolaire. La relation d'amitié s'apparente au lien de clientélisme puisque, exprimée dans un langage affectif, elle se fonde sur la réciprocité. Néanmoins, elle s'en différencie du fait qu'elle établit une relation horizontale et non verticale, unissant des couples de correspondants de statut équivalent. En effet, l'amicitia, lien particulier moralement contraignant, entraîne une obligation de services mutuels entre égaux et implique des dons mutuels et des services réciproques. L'amitié, c'est ce sentiment d'intérêt, cet affectus de l'affectus pour reprendre le jeu de mots qui remonte à Saint Augustin. Cela se traduit concrètement

\footnotetext{
${ }^{66}$ Seule une lettre adressée à une femme porte sur la gestion d'affaires privées en France. Cette lettre est un moyen pour l'époux de se tenir informé sur l'état de ses vignes : «Je sauroys voulumptiers si nos vignes sont belles de par dela, car j'é intencion, à l'ayde de Dieu, que nous ferons vendanges ceste année. », Ibid, p. 13-14.

${ }^{67}$ «Si vous entendez parler de quelque vacance, avertissez m'en, car je voudrais faire avoir à mon fils tant office que benefice, aubeyne ou autre chose. », Ibid, p. 36.

${ }_{68}^{68}$ «Je suis dans la compagnie de Monseigneur de Montpensier, grace à l'ecuyer de Charenault à qui je doit 80 livres. Vouldriez vous les rendre à sa femme ?», Ibid, p. 18.

${ }^{69}$ «Monsieur de saint Legyr [...] vostre fils Louys [...] avet besoin de ung grant cheval ; yl m'a requys que je l'an voussysse secouryr; je l'ay fayt par l'amour de vous comme vous pourés veoyr par les lectres que je croy qui vous an escript: la some du dit cheval se monte IIIXX frans. Je vous suplie qu'il vous plesse de bailler la dyte some à ma famme [...]. », Ibid, p. 17.

${ }^{70}$ Les traités insistent sur l'ordre de la lettre et prône cette composition en cinq points. G. Constable, Letters and letter-collections, Louvain, fasc. 17, 1976, p. 16 sq.
} 
dans les lettres de gentilshommes: par la narratio, le destinateur offre à un correspondant la contrepartie, d'une loyauté égale, à sa supplique ou petitio. Dès lors, la lettre de nouvelles repose bien sur la transmission d'une information et sur la sommation d'une prestation.

\section{Dictare et ses enjeux : l'ambivalence de la recommandation}

Au-delà de l'instrumentalisation de l'information, autrement dit des deux démarches sur lesquelles se fondent les lettres de nouvelles - offrir et demander les missives des gentilshommes sont des lettres de recommandation. Dans ces documents, la requête d'être recommandé auprès de leurs amis est constante. D'après Furetière, la «recommandation » se traduit en termes de civilité : faire sa recommandation à quelqu'un revient à lui témoigner que l'on se souvient de lui. La lettre particulière, en effet, permet à un couple de correspondants, liés entre eux par la parenté ou l'affection, de tisser des relations privées. La lettre est une re-création du réel, un intermédiaire irremplaçable entre la présence et l'absence. Ainsi les lettres de nouvelles présentifient les gentilshommes au sein de leur réseau social. Ce concept de la lettre conçue comme un sermo absentium ou discours de l'absent est bien connu, mais il gagnerait à être compris en termes anthropologiques. La lettre répond en effet à deux désirs, celui d'informer et celui d'être informé. Au delà des bienfaits que procurent les nouvelles, ce souci d'être au courant ou de tenir un individu au courant est particulièrement intéressant : être averti est nécessaire pour se sentir appartenir à une communauté. La présentification du destinateur ne saurait alors se comprendre sans faire référence à la conception de la sociabilité qui ordonne le groupe social.

La recommandation relève également d'une autre acceptation que celle de représentation, à savoir le conseil ou l'avis. Ce second sens exprime toute l'ambivalence du terme dictare et l'enjeu que recouvre la lettre de nouvelles. À l'origine, le terme de dictare signifie prendre sous la dictée un discours et l'organiser. Puis, il a pris le sens d'écrire, de rédiger des actes juridiques et des lettres officielles. Enfin, il a pris le sens de recommander au sens de conseiller, de prescrire $^{71}$.

Par le recours à l'art épistolaire, la distance imposée par la guerre entre les deux «amis » n'entrave pas le réseau d'amitié en lequel s'ordonne la sociabilité du groupe social. Le réseau est géographiquement dilaté, mais la sociabilité perdure. Justement, les lettres des gentilshommes abondent d'indices attestant cette permanence. Il n'est pas rare qu'un destinateur intervienne dans la conclusion d'une union matrimoniale. Par exemple, la missive de G. Popillart à son frère poursuit une discussion débutée de vive voix à propos du projet de mariage de sa nièce avec le

\footnotetext{
${ }^{71}$ Alain Boureau souligne encore un autre emploi du terme dictare, un emploi extensif et mystique de la lettre. En effet, la forme épistolaire a été adoptée par le Christianisme dans le Nouveau Testament, puis dans la liturgie, instaurant une sorte de communication épistolaire avec le sacré. A. Boureau, «La norme épistolaire, une invention médiévale», La correspondance : les usages de la lettre au XIX siècle, dir. R. Chartier, Paris, Fayard, 1991, chap. 2, p. 127-157.
} 
fils de son correspondant, mariage dont il se réjouit ${ }^{72}$. De même, dans une lettre à sa sœur Anne de Tonnere, un gentilhomme préconise à sa cousine, à propos de laquelle court la rumeur de son mariage prochain, de ne pas précipiter sa décision et d'«atendre le retour [des troupes françaises] car il [lui] semble que il y a yci biaucoup de gens qui n'en sont pas contens. $\rangle^{73}$. Les gentilshommes alimentent également leur relation d'amitié par l'envoi de cadeaux qu'ils prennent soin de notifier à leurs correspondants. Ainsi, certains destinateurs les avertissent de l'expédition par voie maritime, prétendument plus sûres, de vêtements, de vin italien, de livre ou de coffrets remplis de bagues soit autant d'objets reflétant la culture italienne dans son raffinement ${ }^{74}$.

Tant l'échange épistolaire que l'envoi de cadeaux est un instrument pour renforcer les liens d'amitié au sein d'un réseau social donné.

Ces lettres de nouvelles unissant des correspondants d'une même couche sociale représentent une source privilégiée pour tenter d'appréhender, d'une part, les mécanismes de la rumeur et, d'autre part, l'instrumentalisation de l'information et de la lettre dans les relations interpersonnelles au sein d'un réseau social. Si ces missives s'inscrivent dans une typologie du genre épistolaire définie notamment par Boncompagno - «les lettres de nouvelles échangées par des amis en temps de guerre »-, les gentilshommes se jouent de l'habilité de la forme réglementée des lettres pour maintenir un apport d'amitié et de proximité. L'instrumentalisation de l'information ne saurait se comprendre sans faire référence au concept de l'amicitia, construit par la circulation de prestations réciproques. Les nouvelles sur le quotidien des troupes et sur l'état de santé des hommes ne sont que les pendants des requêtes de bienfaits, moraux et matériels. De surcroît, derrière ce paravent d'un discours présentifiant, la lettre permet au destinateur d'alimenter et de renforcer sa relation d'amitié en lui proposant de concrètement prendre part à la sociabilité de son réseau social. Ces lettres ne sont nullement des liens ténus qui soutiennent simplement la proximité des gentilshommes avec leur groupe : elles les mettent en jeu dans une dimension cruciale.

Même si le public de ces lettres manuscrites ne se limite pas à leurs seuls destinataires, englobant aussi leur entourage auquel on rapporte de vive voix les nouvelles transalpines, l'imprimerie a joué un rôle déterminant dans la massification de la diffusion de ces informations. En effet, d'autres lettres de nouvelles adressées depuis l'Italie par des hommes de l'armée de Charles VIII sont mises en imprimé essentiellement à Paris, à Lyon et à Rouen, sous forme de «pièces gothiques $»^{75}$. Ce

\footnotetext{
${ }^{72}$ « J'ay les lettres que m'avez escriptes, faisant mention que vouldriés bien traicter le mariage de ma nyepce et de vostre filz ainsi que pieça vous et moy parlasmes ensemble », A. Cutolo, op. cit., p. 40.

${ }^{73}$ Ibid, p. 40-41.

${ }^{74}$ « J'ay mis dans la galliace de France qui s'en va cent livres de lins et ung coffret plain de petits follies [...] et envoie aussi ung coffret plein de bagues », Ibid, p. 55, 38 .

${ }^{75}$ Dès les années 1480, ces opuscules constitués de quelques feuillets et agrémentés de gravures sur bois colportent régulièrement auprès de lecteurs-auditeurs des nouvelles concernant le roi de France et le royaume - les cérémonies d'entrée solennelle - et les événements de l'expédition italienne - batailles, assauts et victoires royales -, afin d'en
} 
sont des missives de combattants, rédigées «au camp ", comparables à celles des gentilshommes dans leur forme mais davantage centrées sur les événements militaires. Cela s'explique aisément la coïncidence de leur contexte d'écriture avec les temps forts de la campagne. Hélas, la confrontation des auteurs de ces deux correspondances, les gentilshommes et les combattants, se heurte au souci des compositeurs des "pièces gothiques»de rendre anonymes les lettres mises en imprimé : à la manière des lettres-modèles des artes dictamini, ils éliminent tout contenu personnel. Mais, de ce fait, les combattants se font en quelque sorte les « yeux » de la communauté de France en exposant ce qu'ils ont vu ou vécu ${ }^{76}$. Leurs témoignages, dépourvus de toutes traces d'individualisation, permettent aux lecteurs-auditeurs de s'identifier à ces hommes, de s'approprier leurs témoignages et de s'affirmer comme membres à part entière de la communauté de France.

L'imprimerie densifie alors le réseau social initial alimenté par ces lettres. Accompagnées d'images, de pièces en vers ou de lettres royales, les lettres de nouvelles sont désormais instrumentalisées à des fins politiques ou de propagande.

Marion Pouspin

EHESS

conserver la mémoire pour la postérité et d'instiller ou entretenir dans les esprits des contemporains une image toujours favorable à la monarchie de France. M. Pouspin, «Les "pièces d'actualité politique » françaises : événements, représentations et mémoire », Images re-vues, 5, 2008. http://www.imagesrevues.org/Article_Archive.php?id_article=34.

${ }^{76}$ Ces lettres de combattants sont authentiques: certaines lettres mises en imprimé sont conservées dans leur version manuscrite à la bibliothèque de Nantes sous les cotes 41030 et 41110 
ANNEXES

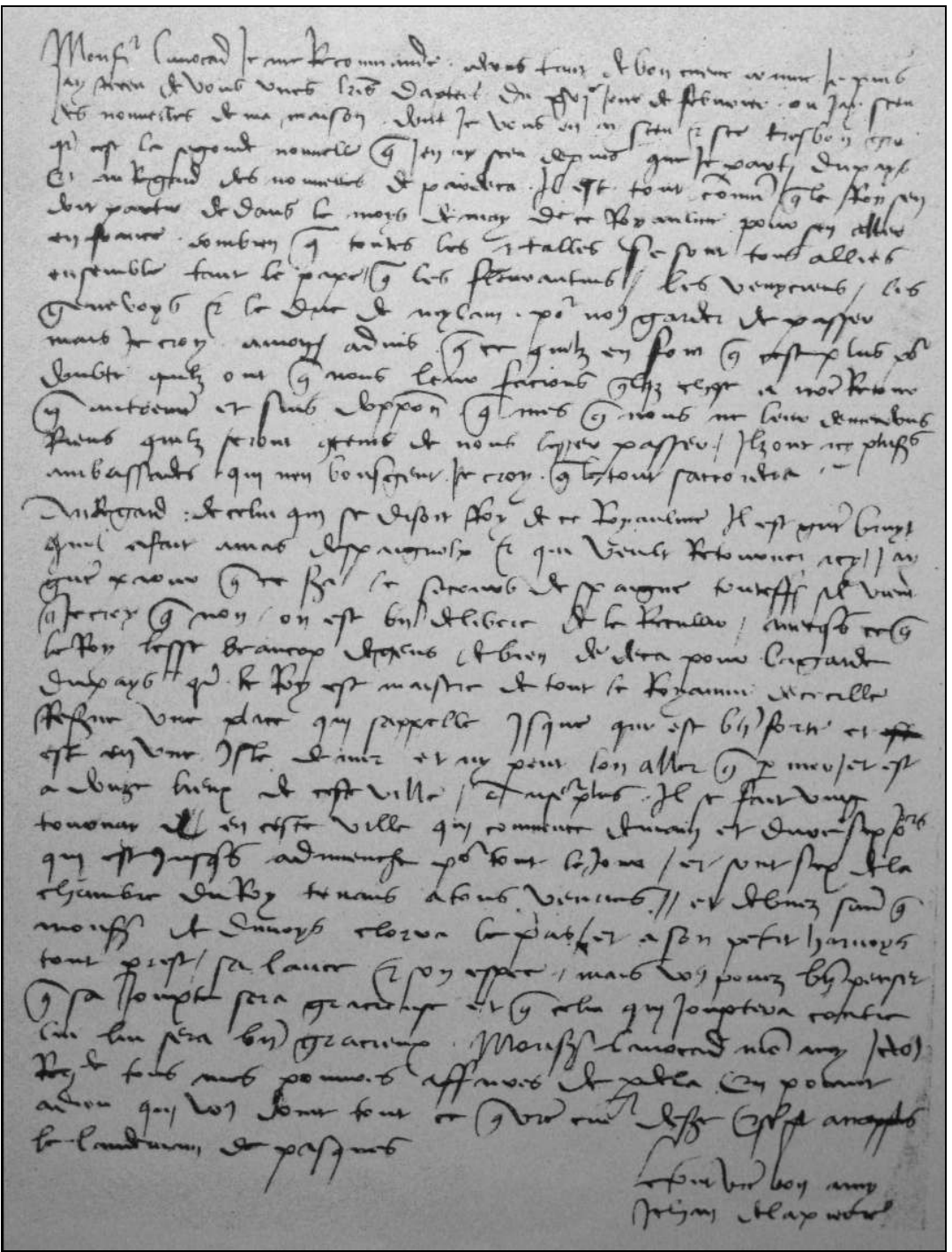


LeTtre DE JEAN DE LAPIERRE A RENAUd SOHIER,

PROCUREUR DU MARECHAL DE GIE (NAPLES, 20 FEVRIER 1495)

A. Cutolo, Op. cit., p. 10 bis.

Lettre de Jean de Lapierre à Renaud Sohier, procureur du maréchal de Gié (Naples, 20 avril 1495), l'informant du retour imminent du roi dans le royaume de France, puis de la rumeur du renfort espagnol à la Ligue italienne et, enfin, des joutes auxquelles participe le maréchal de Gié. [Arch. Milan, Cart. 250]

«Monsieur l'avocad, je me recomnande à vous tout de bon cueur comme je puis. / J'ay receu de vous unes lettres daptées du XVI jour de febvrier ou j'ay sceu / des nouvelles de ma maison dont je vous en ay sceu et scé tres bon gré / quar c'est la seconde nouvelle que je vous ay sceu depuis que je parti du pays. / Et au regard des nouvelles de par deca, il est tout commun que le Roy s'en / doit partir dedans le moys de may de ce Royaulme pour s'en aller/en France, combien que toutes les Italles se sont alliés / ensemble tant le pape, que les Fleurantins, les Venyciens, les / Genevoys et le duc de Mylan, pour nous garder de passer, / mais je croy, à mons advis, que ce qu'ilz en font, que c'est plus pour / doubte qu'ilz ont que nous leur facions quelquez chose à nostre retour / que autrement, et suis d'oppinion que mes que nous ne leur demendons / riens, qu'ilz seront contems de nous lesser passer. Ilz ont icy plusieurs / ambassades qui n'en bousgent. Je croy que le tout s'accordera. // Au regart de celui qui se disoit Roy de ce royaulme, il est grant bruyt / qu'il a fait amas d'Espaignolx et qui veult retourner icy. J'ay / grant paour que ce sera le secours d'Espaigne. Touteffoys s'il vient, / que je croy que non, on est bien deliberé de le reculler. Avecques ce que / le Roy lesse beaucop de gens de bien de deca pour la garde / du pays, quar le Roy est maistre de tout le royaume de Cecille, / reserve une place qui s'appelle Isque, qui est bien forte et [rature sur « est »] / est en une isle de mer et n'y peut l'on aller que par mer, et est / à douze lieux de ceste ville. Au surplus, il se fait ung / tournoy [rature] en ceste ville qui comnence demain et dure six jours, / qui est jusques à dimenche, pour tout le jour, et sont six de la / chambre $\mathrm{du}$ Roy tenans à tous venans, et debvez savoir que / monsieur de Dunoys clorra le pas, et à son petit harnoys / tout prest, sa lance et son espée, mais vous povez bien penser / que sa jouxte sera gracieuse et que celui qui jouxtera contre / lui lui sera bien gracieux. Monsieur l'avocad, mon amy, je vous / recommande tous mes pouvres affaires de par dela. En priant / à Dieu qui vous doint tout ce que vostre cueur desir. Escrip à Napples, / le landemain de pasques. // Le tout vostre amy // Jehan de Lapierre. / » 